\title{
THE RADIUS AND MODULUS OF $n$-VALENCE FOR ANALYTIC FUNCTIONS WHOSE FIRST $n-1$ DERIVATIVES VANISH AT A POINT
}

\author{
LYNN H. LOOMIS
}

The principal result of this note is the determination of the precise radius and modulus of $n$-valence for the class of functions $f(z)=z^{n}+a_{n+1} z^{n+1}+\cdots$ analytic and less than or equal to $M$ in modulus in $|z| \leqq 1$. This result readily leads to the radius and modulus of $n$-valence for the more general class of functions $f(z)=a z^{n}+a_{n+1} z^{n+1}+\cdots$ analytic and less than or equal to $M$ in modulus in $|z| \leqq R$. Finally, we note certain approximations which rather naturally suggest themselves in a search for more easily calculable constants.

We consider only expansions about the origin of functions $f(z)$ with $f(0)=0$, the generalization to expansions about $a$ of functions $f(z)$ with $f(a)=b$ being obvious. Each circle mentioned will be understood to have the origin ( $w=0$ or $z=0$ ) as center. The phrases radius of $n$-valence and modulus of $n$-valence, which usually refer to a class of functions, will also be used with reference to a single function. The radius of $n$-valence of the function $f(z)$ is the radius of the largest circle within which $f(z)$ assumes no value more than $n$ times, and assumes at least one value $n$ times. The modulus of $n$-valence of $f(z)$ is the radius of the largest circle of which the interior is covered exactly $n$ times by the map under $f(z)$ of $|z|<\rho$, where $\rho$ is the above radius of $n$-valence. Consider now one of the classes defined above. It is obvious that for each function $w=f(z)$ of the class there is a neighborhood of $z=0$ in which the function assumes no value more than $n$ times, and assumes exactly $n$ times every value in a sufficiently small neighborhood of $w=0$. The radius of $n$-valence $\rho_{n}$ of the class is the radius of the largest circle within which no function of the class assumes a value more than $n$ times. The modulus of $n$-valence $m_{n}$ of the class is the radius of the largest circle of which the interior is covered exactly $n$ times by the map of $|z|<\rho_{n}$ under every function of the class.

TheOREM. Consider the class of functions $f(z)=z^{n}+a_{n+1} z^{n+1}+\cdots$ analytic and less than or equal to $M(M>1)^{1}$ in modulus in $|z| \leqq 1$,

\footnotetext{
1 The restriction to $M>1$ is necessary. By the Cauchy coefficient inequality, $M \geqq 1$, and if $M=1$ the class consists of the single function $f(z)=z^{n}$ for which the theorem is false.
} 
where $n$ and $M$ are the constants of the class. The radius $\rho_{n}$ and the modulus $m_{n}$ of $n$-valence of the class are given by

where

$$
m_{n}=\frac{M \rho_{n}^{n}\left(1-M \rho_{n}\right)}{M-\rho_{n}}, \quad \rho_{n}=M_{n}-\left(M_{n}^{2}-1\right)^{1 / 2},
$$

$$
M_{n}=\frac{1}{2}\left[\left(1+\frac{1}{n}\right) M+\left(1-\frac{1}{n}\right) \frac{1}{M}\right] .
$$

The case for $n=1$ is completely treated in the literature. The results are due to Landau and Dieudonné. ${ }^{2}$ The values of $\rho_{1}$ and $m_{1}$ are

$$
\rho_{1}=M-\left(M^{2}-1\right)^{1 / 2}, \quad m_{1}=M \rho_{1}^{2}=M \rho_{1} \frac{\left(1-M \rho_{1}\right)}{M-\rho_{1}} .
$$

The following proof for general $n$ consists of

(a) a proof that every function of the class is $n$-valent and covers $|w|<m_{n}$ exactly $n$ times in $|z|<\rho_{n}$, and

(b) the exhibition of a single function of the class for which $\rho_{n}$ and $m_{n}$ are respectively the radius and modulus of $n$-valence.

We employ the device which Dieudonne used to find the radius of star-shapedness in the case $n=1$ (Montel, loc. cit., p. 94), and then apply a theorem due to $\mathrm{S}$. Ozaki, ${ }^{3}$ which states that if $f(z)$ is analytic in $|z| \leqq r$ and has $n$ zeros there, none on the circumference, and if for some real $\alpha, \Re\left[e^{i \alpha} z f^{\prime}(z) / f(z)\right]>0$ on $|z|=r$, then $f(z)$ is $n$-valent in the circle $|z|<r$.

Consider

$$
g(z)=f(z) / z^{n}=1+a_{n+1} z+\cdots .
$$

Since $g(z)$ is analytic and less than or equal to $M$ in modulus in $|z| \leqq 1$ with $g(0)=1$, the following inequalities, results of the Schwarz lemma, are valid (Montel, loc. cit., p. 91):

$$
\begin{gathered}
|g(z)| \geqq \frac{M(1-M r)}{M-r}, \\
\left|g^{\prime}(z)\right| \leqq \frac{M^{2}-|g(z)|^{2}}{M\left(1-r^{2}\right)},
\end{gathered}
$$

${ }^{2}$ See Montel, Leçons sur les Fonctions Univalentes ou Multivalentes, 1933, pp. 9095. This book contains a convenient collection of material relating to this paper, and we shall often refer to it.

${ }^{3} \mathrm{~S}$. Ozaki, Some remarks on the univalency and multivalency of functions, Science Reports of Tokyo Bunrika Daigaku, section A, 2, no. 32, 1934, pp. 41-55. 
where $r=|z|<1 / M$. From (1) and (2),

$$
\left|\frac{z g^{\prime}(z)}{g(z)}\right| \leqq \frac{r\left(M^{2}-1\right)}{(M-r)(1-M r)}
$$

if $r<1 / M$. But we have

$$
\begin{aligned}
g^{\prime}(z) & =\frac{z^{n} f^{\prime}(z)-n z^{n-1} f(z)}{z^{2 n}}, \\
\frac{z f^{\prime}(z)}{f(z)} & =\frac{z g^{\prime}(z)}{g(z)}+n .
\end{aligned}
$$

By (3) and (4) $\Re\left[z f^{\prime}(z) / f(z)\right]>0$ whenever

$$
n>\frac{r\left(M^{2}-1\right)}{(M-r)(1-M r)} \text {. }
$$

The latter inequality reduces, for $r<1 / M$, to

$$
r^{2}-\left[\left(1+\frac{1}{n}\right) M+\left(1-\frac{1}{n}\right) \frac{1}{M}\right] r+1>0
$$

which is satisfied (factoring the left member) if

$$
r<M_{n}-\left(M_{n}^{2}-1\right)^{1 / 2}=\rho_{n},
$$

where

$$
M_{n}=\frac{1}{2}\left[\left(1+\frac{1}{n}\right) M+\left(1-\frac{1}{n}\right) \frac{1}{M}\right] .
$$

Now $\rho_{n}<1 / M$, for we shall see that $\rho_{n}$ is the smallest positive zero of the derivative of

$$
M x^{n}(1-M x) /(M-x)
$$

and this lies between 0 and $1 / M$. But from (1)

$$
|f(z)| \geqq \frac{M r^{n}(1-M r)}{M-r} .
$$

Hence $f(z)$ has precisely $n$ zeros in $|z|<r$ and none on the boundary $|z|=r$, for every $r \leqq \rho_{n}$. Then:

(1) By (5), $|f(z)| \geqq m_{n}$ on $|z|=\rho_{n}$. Thus, by Rouché's theorem, $f(z)$ assumes exactly $n$ times in $|z|<\rho_{n}$ every value $w$ with $|w|<m_{n}$.

(2) We have seen that $\Re\left[z f^{\prime}(z) / f(z)\right]>0$ on $|z|=r<\rho_{n}$.

Thus, by the theorem of Ozaki noted above, $f(z)$ is $n$-valent in the 
region $|z|<r$ for every $r<\rho_{n}$, and hence $n$-valent in $|z|<\rho_{n}$. This completes the proof of (a).

It follows from the Schwarz lemma that equality can occur in (1) for a point in $0<|z|<1$ only if $g(z)$ is of the form

$$
g(z)=\frac{M\left(1-M e^{i \theta} z\right)}{M-e^{i \theta} z} .
$$

We obtain the simplest function with $e^{i \theta}=1$, giving

$$
f_{0}(z)=\frac{M z^{n}(1-M z)}{M-z} .
$$

This function is of the class considered, and has $\rho_{n}$ and $m_{n}$ for its radius and modulus of $n$-valence. For,

$$
f_{0}^{\prime}(z)=\frac{M^{2} n z^{n-1}}{(M-z)^{2}}\left\{z^{2}-\left[\left(1+\frac{1}{n}\right) M+\left(1-\frac{1}{n}\right) \frac{1}{M}\right] z+1\right\},
$$

and it is evident that the zero of $f_{0}^{\prime}(z)$ nearest the origin (except $z=0$ itself) is precisely $\rho_{n}$. Also

$$
f_{0}\left(\rho_{n}\right)=\frac{M \rho_{n}^{n}\left(1-M \rho_{n}\right)}{M-\rho_{n}}=m_{n} .
$$

Thus these constants are respectively the radius and modulus of $n$ valence for this function, and hence by (a) for the class considered in the theorem.

Corollary 1. Consider the class of functions $f(z)=a z^{n}+a_{n+1} z^{n+1}$ $+\cdots$ analytic and less than or equal to $M\left(M>|a| R^{n}\right)$ in modulus in $|z| \leqq R$, the constants of the class being $|a|(\neq 0), n, M$ and $R$. The radius $\rho_{n}$ and the modulus $m_{n}$ of n-valence of the class are given by

$$
\rho_{n}=R \sigma, \quad m_{n}=\frac{M \sigma^{n}\left(|a| R^{n}-M \sigma\right)}{M-|a| R^{n} \sigma},
$$

where $\sigma$ is defined by

$$
\begin{aligned}
\sigma & =M_{n}-\left(M_{n}^{2}-1\right)^{1 / 2} \\
M_{n} & =\frac{1}{2}\left[\left(1+\frac{1}{n}\right) \frac{M}{|a| R^{n}}+\left(1-\frac{1}{n}\right) \frac{|a| R^{n}}{M}\right] .
\end{aligned}
$$

This corollary follows immediately from the theorem upon considering the function $g(z)=f(R z) / a R^{n}$. 
The results on the modulus of $n$-valence relate to a result due to Walsh and Seidel, ${ }^{4}$ who do not assume $f^{\prime}(0)=\cdots=f^{(n-1)}(0)=0$, but who, on the other hand, do not obtain the sharp inequality.

Corollary 2. For the class of functions of Corollary 1

$$
\rho_{n}>\frac{|a| R^{n+1}}{2 M}, \quad m_{n}>M\left(\frac{|a| R^{n}}{2 M}\right)^{n+1} .
$$

For, $M /|a| R^{n}>1$, from which it follows that $M /|a| R^{n} \geqq M_{n}>1$. Therefore, since

$$
M_{n}-\left(M_{n}^{2}-1\right)^{1 / 2}=\frac{1}{M_{n}+\left(M_{n}^{2}-1\right)^{1 / 2}}>\frac{1}{2 M_{n}},
$$

we find that

$$
\frac{|a| R^{n+1}}{2 M}<\rho_{n} .
$$

Moreover, if $f(z)=M z^{n}\left(|a| R^{n+1}-M z\right) /\left(M R^{n+1}-|a| R^{2 n} z\right)$, then $f\left(\rho_{n}\right)$ $=m_{n}, f^{\prime}\left(\rho_{n}\right)=0, f\left(|a| R^{n+1} / 2 M\right)<m_{n}$ by (6), and the last inequality easily gives

$$
M\left(\frac{|a| R^{n}}{2 M}\right)^{n+1}<m_{n}
$$

But (6) and (7) constitute the corollary.

The inequality (7) is an improvement over an approximation due to Privaloff, ${ }^{5}$ which states that the image of the circle $|z|<R$ under the function $w=f(z)$ of the class of Corollary 1 covers at least $n$ times the circle

$$
|w|<\frac{8}{3} M\left(\frac{R^{n}|a|}{4 M}\right)^{n+1}
$$

For,

$$
M\left(\frac{|a| R^{n}}{2 M}\right)^{n+1}>\frac{8}{3} M\left(\frac{|a| R^{n}}{4 M}\right)^{n+1},
$$

and by (7), the circle $|w|<M\left(|a| R^{n} / 2 M\right)^{n+1}$ is covered exactly $n$ times by the image of $|z|<\rho_{n}$ and hence at least $n$ times by that of $|z|<R$.

4 Walsh and Seidel, On the derivatives of functions analytic in the unit circle, Proceedings of the National Academy of Sciences, vol. 24 (1938), pp. 337-340.

${ }^{5} \mathrm{~J}$. Privaloff, Sur un théorème de $M$. Bloch, Recueil Mathématique de Moscou, vol. 35 (1928), pp. 111-121. 
We conclude with a few miscellaneous remarks.

1. The modulus of $n$-valence for the class of the theorem is also the radius of the largest circle within which converge all the power series (in $w^{1 / n}$ ) of the inverse functions.

2. It is evident from the proof of part (b) of the theorem that the radius and modulus of $n$-valence for the function $f(z)$ are $\rho_{n}$ and $m_{n}$ only if

$$
f(z)=\frac{M z^{n}\left(1-M e^{i \theta} z\right)}{M-e^{i \theta} z}
$$

for some real $\theta$. For any other function of the class the radius and modulus of $n$-valence are greater, respectively, than $\rho_{n}$ and $m_{n}$.

3. The inequalities $\leqq M$, $\leqq 1$ of the theorem may be replaced by $<M,<1$ without affecting the validity of the work.

4. It is easily seen that the equations of Corollary 1 give, for instance, the radius of $n$-valence for the class of functions of the form $f(z)=a_{f} z^{n}+a_{n+1} z^{n+1}+\cdots$ analytic and less than or equal to $M_{f}$ in modulus in $|z| \leqq R$, including just those functions for which $M_{f} /\left|a_{f}\right|$ is less than some preassigned bound $(M /|a|$ of the equations of the corollary). No modulus of $n$-valence exists for this class.

Harvard University 